\title{
Connections: can the 20th century coronary heart disease epidemic reveal something about the 1918 influenza lethality?
}

\author{
M.I. Azambuja \\ Programa de Pós-Graduação em Epidemiologia, Departamento de Medicina Social, Universidade \\ Federal do Rio Grande do Sul, Porto Alegre, RS, Brasil
}

Correspondence to: M.I. Azambuja, Programa de Pós-Graduação em Epidemiologia, Departamento de Medicina Social, UFRGS, Rua Ramiro Barcelos, 2600, 4/420, 90035-003 Porto Alegre, RS, Brasil

Fax: +55-51-3308-5291. E-mail: miazambuja@terra.com.br

This essay proposes that the ecologic association shown between the 20th century coronary heart disease epidemic and the 1918 influenza pandemic could shed light on the mechanism associated with the high lethality of the latter. It suggests that an autoimmune interference at the apoB-LDL interface could explain both hypercholesterolemia and inflammation (through interference with the cellular metabolism of arachidonic acid). Autoimmune inflammation, then, would explain the 1950s-60s acute coronary events (coronary thrombosis upon influenza re-infection) and the respiratory failure seen among young adults in 1918. This hypothesis also argues that the lethality of the 1918 pandemic may have not depended so much on the 1918 virus as on an immune vulnerability to it, possibly resulting from an earlier priming of cohorts born around 1890 by the 1890 influenza pandemic virus.

Key words: Respiratory distress syndrome; Autoimmunity; Influenza; Coronary disease; Disease susceptibility; Disease outbreaks

Received April 13, 2007. Accepted September 17, 2007

We may be living in revolutionary times in the field of biological sciences. As stated by Goldenfeld and Woese (1) referring to the evidence of horizontal gene-swapping within microbe collectives, "molecular reductionism that dominated the 20th century biology will be superseded by an interdisciplinary approach that embraces collective phenomena (p. 369)". Also the growing recognition of the coconstruction of both life and its environment at the molecular (2), organism (3-5) and population (6) levels, increasingly frames such collective phenomena as contextual. According to Gould (6), the events of our complex natural world always result from particular historical sequences that "unfurl along the pathways actually followed, rather than along any of the myriad equally plausible alternatives (p. 36)". Science always mistrusted and downgraded contingent events. The experimental methodology and the "laws of nature" type of explanations assumed such a paradigmatic strength in science as to be considered the sole representatives of the true scientific endeavor. But, according to Gould, the contingency's domain needs to be recognized in its true dimension: one as broad and important as anything deducible from natural law. "Contingent events, though unpredictable at the onset of a sequence, are as explainable as any other phenomena after they occur. The explanations as contingent rather than lawbased do require a knowledge of the particular historical sequence that generated the result, for such resolutions must be in the narrative rather than deductive model $(6, p$. 36)".

Traditional epidemiology - the one practiced until the 1940s-50s and defined by Kuller (7) as "the study of epidemics and their prevention (p. 1051)" - was conceived 
as a way to investigate contingent events, with the purpose of preventing similar occurrences in the future: "Epidemiology was 1) the study of epidemics (contingent events) in relation to time, place and person; 2) built on a strong biological rationale, and 3) the basic science of public health (7, p. 1051)".

Epidemics are unique events with respect not only to the environmental challenge initiating them, but also to the circumstantial pattern of resistance/vulnerability (for the lack of a better concept) of the exposed population, built during the course of all previous nature-nurture interactions taking place on and across its several levels of organization (molecular, individual and collective) along time (history) (8).

The 1918 influenza pandemic was unusually severe, causing about 40 million deaths around the world. Its high level of lethality has been attributed to the virus itself $(9,10)$. However, this interpretation is not consistent with the contextual nature of a new biology. If context is indeed important, the 1918 phenomenon must have resulted from a convergence between the virus and a particular pattern of population vulnerability to it. Supporting this claim is the unusual concentration of high rates of lethality among young adults found during that period (11). Since we would not know how to identify vulnerability to the virus at the individual level, we would need to look for clues as to its distribution at the only place they might be found, i.e., at the population level (12).

Based on this rationale, it would make sense to investigate the mechanism resulting in the 1918 influenza lethality by looking at another collective phenomenon proposed to be associated with the vulnerability of the 1918 population to the influenza virus, i.e., the 20th century coronary heart disease (CHD) epidemic (8,13-15).

Wade Hampton Frost (16), a leading epidemiologist of the US Public Health Service, wrote in 1927: "The opinion is more or less prevalent that inferences based upon epidemiological argument cannot be truly conclusive, because the evidence is purely circumstantial. Such opinion has frequently failed to take account of the whole mass of evidence, and to follow the argument which is necessary built up step by step in a somewhat complex and perhaps tedious way. Given sufficient scope and accuracy of observations, a conclusion as to the nature and spread of a disease may often be established quite firmly by circumstantial evidence well in advance of experimental confirmation... The weakness in conclusions drawn from circumstantial studies is usually chargeable not to basic defects in the methods of investigation, but more often to paucity or inaccuracy of data, or faults of logic in their interpretation (16, p. 190)".
Following Frost's lead, the idea is that the clues regarding this epidemiologic association together with a strong biologic rationale - drawn, by analogy, from evidence reported in the literature - could shed light on the mechanism responsible for the influenza lethality in advance of experimental confirmation. It is left to the reader to judge the results of this enterprise.

It was first proposed in 1994 (13) that a cohort association existed between rates of vulnerability to influenza deaths in 1918 and CHD mortality among survivors from those vulnerable birth cohorts. According to this hypothesis, hypercholesterolemia - the hallmark of $\mathrm{CHD}$ cases in the 1950s and 60s - may have been an effect secondary to the influenza priming of 1918 vulnerable individuals for late development of CHD. CHD deaths, however, were probably associated with bursts of auto-immune endothelial inflammation and thrombosis associated with influenza re-infections $(8,14,15)$.

Mimicry between the amino acid sequences involved in cell attachment of the viral hemagglutinin and those of apolipoprotein B (apoB) involved in the low-density lipoproteins (LDL) binding to high affinity LDL receptors (LDLR) has been described in some strains of the influenza virus (17).

An autoimmune interference at the apoB-LDLR interface might explain the development of hypercholesterolemia. In familial hypercholesterolemia, raised serum LDL cholesterol levels result from an interference in the LDLR pathway, commonly caused by a loss of function mutation in the LDLR gene or by a mutation in the gene encoding apoB (18).

Also, cross-reactive auto-antibodies directed against the apoB-LDLR interface could lead to sub-endothelial coaccumulation of lipids and immune products, and result in lipid peroxidation. This sequence of events has been fully demonstrated in cases of Heymann nephritis, an autoimmune disease in which the main auto-antibodies target (megalin/gp330) is also a member of the LDLR family (19).

Thus, it would be possible that, in vulnerable individuals, cross-reactive auto-antibodies (and T cells) diverted to the apoB-LDLR interface, first elicited by the 1918 influenza infection and recalled upon influenza reinfections of 1918 primed individuals, resulted in elevated serum cholesterol levels (aggravated by high-fat diets) and lipid deposits co-localized at sites of viral penetration: the left side of the heart, coronary arteries and the aortic arch $(8,14,15)$.

But what would the mechanism initiating the autoimmune endothelial inflammation and thrombosis resulting in the high CHD lethality observed during the 1950s and 1960s influenza epidemics be? And could it give us a clue 
as to the mechanism responsible for the high lethality of the 1918 influenza pandemic?

The recent discussion about untoward cardiovascular effects of selective anti-inflammatory drugs has taught us about an important group of inflammatory mediators associated with vascular homeostasis, i.e., the cyclooxygenase (COX) products (20). COX is the rate-limiting enzyme in the synthesis of prostaglandins (PG) from free arachidonic acid (AA). It exists as two isoforms, COX-1 (mostly constitutive) and COX-2 (mostly inducible in pathologic situations) (20).

In 1988, Krone et al. (21) showed that PGE1, PGE2, and prostacyclin (PGI2) affected the LDLR activity and cholesterol synthesis in isolated mononuclear leukocytes. In 2002, Smith et al. (22) showed that, in cultured endothelial cells, PGI2 formation is regulated by LDL cholesterol levels. Both findings reinforce the suggestion made by Salbach et al. (23) in 1991 of a new role for the LDLR, the regulation of cellular levels of free $A A$, and hence, of $P G$ synthesis.

The LDLR AA pathway does appear to couple directly to the PGH synthase reaction, but not to the 5-lipoxygenase (5-LOX) reaction (24). Thus, upon re-infection, an interference on this pathway could result in a LOX-COX products imbalance. Interestingly, polymorphism studies of the gene ALOX5AP (which encodes the 5-LOX-activating protein) have shown that variants of this gene are associated with twice the risk of developing myocardial infarction and stroke. These high-risk variants are associated with increased production of leukotriene B4 - a key product of the 5-LOX pathway (25). It is possible that any imbalance in favor of 5-LOX products, irrespective of its cause, increases the risk of vascular inflammation and thrombosis.

If the experimental evidence suggests that: a) LDLR has a role both in the cellular cholesterol metabolism $(18,19)$ and in the regulation of PG synthesis $(21-23)$, b) cross-reactive autoimmunity may target members of the LDLR family (19), and c) mimicry may exist between amino acid sequences involved in cell attachment of the viral hemagglutinin and those of apoB involved in LDL binding to high affinity LDLR (17), then a cross-reactive autoimmune response that targets the LDLR-apoB interface could explain not only the cholesterol-related features of the atherosclerotic process, but also the thrombotic component of the atheroma, which was proposed by Morris $(26,27)$ in 1951 to be the main factor responsible for the CHD-related events.

Furthermore, as we will see, the same interference could be implicated in the immunopathogenic response to the 1918 influenza infection. Recently, COX-1 and COX-2 enzyme concentrations were shown to have significant effects on the host response to influenza $A$ viral infection (28). Among other findings, the authors described a marked elevation of lung viral titers in COX-2-l- mice relative to wild-type and COX-1/- mice on day 4 of infection (28). This resembles the unabated load of viral antigens in lungs of 1918 virus-infected monkeys by day 6, compared to no viral antigen expression in K173 virus-infected animals, recently described by Kobasa et al. (10). The whole picture of antiviral response dysregulation in macaques infected with the 1918 influenza virus (10) is highly suggestive of an imbalance in COX products expected to modulate the inflammatory process.

Thus, available experimental evidence supports the epidemiologic hypothesis of association between the 1918 influenza lethality and the 20th century CHD mortality. Or, conversely, the (epidemiologic) cohort association shown to exist between the 1918 influenza lethality and the 20th century CHD mortality suggests a common mechanism for both events, i.e, an interference at the apoB-LDL interface, possibly autoimmune and restricted to some particularly vulnerable birth cohorts. Such interference would explain the high serum cholesterol levels characteristic of CHD cases occurring at the height of the CHD epidemic, the thrombosis found in association with sudden unexpected CHD deaths in the 1950s (reinfection-related?), and also the high lethality of the 1918 influenza epidemic.

The cause of the high vulnerability of the 1918 young adults to autoimmunity and high lethality in 1918 and to high CHD mortality in the years to come would still remain unexplained. An interesting possibility would be that their particular cohort vulnerability had been initiated many years earlier, when they first encountered an influenza virus, which, in their case, was possibly the virus associated with the 1890 influenza pandemic. Recently, it was shown that hidden epitopes may emerge after a secondary challenge with an influenza virus, which may switch protective immunity to an alternative antibody-mediated pathway (29). Thus, it would be possible that the high pathogenicity of the influenza infection registered among young adults in 1918 resulted from an immune response that went awry among those individuals originally primed by the 1890 virus.

A historically built vulnerability of particular birth cohorts to death during the 1918 influenza pandemic and, later on, to death from CHD (coronary thrombosis) upon influenza reinfection would be a strong argument in favor of a new biology, understood as a contextual science.

This paper demonstrates the relevance of epidemiology, here understood as a discipline engaged in the study of collective, contextual phenomena, either as a source of 
hypotheses (like the association between the 1918 influenza pandemic and the CHD epidemic, and between the 1918 lethality and earlier priming by the 1890 influenza virus) or as a checking point to which knowledge produced at the individual and sub-individual levels needs to conform (and vice versa). Making connections between the different levels of biologic organization and knowledge within a contextual framework may be the formula needed to escape the 20th century reductionism and to advance biological knowledge.

\section{REFERENCES}

1. Goldenfeld N, Woese C. Biology's next revolution. Nature 2007; 445: 369

2. Kauffman SA. Prolegomenon to a general biology. Ann N Y Acad Sci 2001; 935: 18-36.

3. Lewontin R. The triple helix. Gene, organism and environment. Cambridge: Harvard University Press; 2001.

4. Meaney MJ. Nature, nurture, and the disunity of knowledge. Ann NY Acad Sci 2001; 935: 50-61.

5. Tauber Al. The immune self: theory or metaphor? Cambridge studies in philosophy and biology. New York: Cambridge University Press; 1997.

6. Gould SJ. "What is life?" as a problem in history. In: Murphy MP, O'Neill LAJ (Editors), What is life? The next fifty years. Speculations on the future of biology. Cambridge: Cambridge University Press; 1995. p 25-39.

7. Kuller LH. Epidemiology is the study of "epidemics" and their prevention. Am J Epidemiol 1991; 134: 1051-1056.

8. Azambuja MI, Levins R. Coronary heart disease (CHD) one or several diseases? Changes in the prevalence and features of CHD. Perspect Biol Med 2007; 50: 228-242.

9. Kash JC, Tumpey TM, Proll SC, Carter V, Perwitasari O, Thomas MJ, et al. Genomic analysis of increased host immune and cell death responses induced by 1918 influenza virus. Nature 2006; 443: 578-581.

10. Kobasa D, Jones SM, Shinya K, Kash JC, Copps J, Ebihara $\mathrm{H}$, et al. Aberrant innate immune response in lethal infection of macaques with the 1918 influenza virus. Nature 2007; 445: 319-323.

11. Collins SD. Age and sex incidence of influenza and pneumonia morbidity and mortality in the epidemic of 1928-29 with comparative data for the epidemic of 1918-19. Public Health Rep 1931; 46: 1909-1937.

12. Evans AS. Viruses. In: Schottenfeld D, Fraumeni JF Jr (Editors), Cancer epidemiology and prevention. Philadelphia: W.B. Saunders; 1982. p 364-390.

13. Reinert-Azambuja MI. Influenza pandemic and ischemic heart disease epidemic: cause and effect? Xth International Symposium on Atherosclerosis. Montréal: 1994. p 109-328 [Abstract].

14. Azambuja MI, Duncan BB. Similarities in mortality patterns from influenza in the first half of the 20th century and the rise and fall of ischemic heart disease in the United States: a new hypothesis concerning the coronary heart disease epidemic. Cad Saúde Pública 2002; 18: 557-566.

15. Azambuja MI. Spanish flu and early 20th-century expansion of a coronary heart disease-prone subpopulation. Tex Heart
Inst J 2004; 31: 14-21.

16. Frost WH. Epidemiology. In: Nelson Loose-Leaf System (Editor), Public Health-Preventive Medicine. New York: Thomas Nelson \& Sons; 1927. p 163-190 (quoted in Sartwell PE. The contribution of Wade Hampton Frost. Am J Epidemiol 1976; 104: 386-391, p 390).

17. Pleskov VM, Bannikov Al, Zaitsev I. The receptor-mediated endocytosis of influenza viruses and low-density lipoproteins by tissue cells. Vopr Virusol 1994; 39: 121-125.

18. Soutar AK, Naoumova RP. Mechanisms of disease: genetic causes of familial hypercholesterolemia. Nat Clin Pract Cardiovasc Med 2007; 4: 214-225.

19. Kerjaschki D, Exner M, Ullrich R, Susani M, Curtiss LK, Witztum JL, et al. Pathogenic antibodies inhibit the binding of apolipoproteins to megalin/gp330 in passive Heymann nephritis. J Clin Invest 1997; 100: 2303-2309.

20. Sohn HY, Krotz F. Cyclooxygenase inhibition and atherothrombosis. Curr Drug Targets 2006; 7: 1275-1284.

21. Krone W, Klass A, Nagele H, Behnke B, Greten H. Effects of prostaglandins on LDL receptor activity and cholesterol synthesis in freshly isolated human mononuclear leukocytes. J Lipid Res 1988; 29: 1663-1669.

22. Smith LA, Boutaud O, Breyer M, Morrow JD, Oates JA, Vaughan DE. Cyclooxygenase-2 dependent prostacyclin formation is regulated by low density lipoprotein cholesterol in vitro. Arteriosc Thromb Vasc Biol 2002; 22: 983-988.

23. Salbach PB, Janssen-Timmen U, Blattner C, Ziegler R, Habenicht AJ. A new role for the low density lipoprotein receptor. Z Gastroenterol Verh 1991; 26: 107-109.

24. Janssen-Timmen U, Tomic I, Specht E, Beilecke U, Habenicht AJ. The arachidonic acid cascade, eicosanoids, and signal transduction. Ann N Y Acad Sci 1994; 733: 325334.

25. Helgadottir A, Manolescu A, Thorleifsson G, Gretarsdottir $\mathrm{S}$, Jonsdottir $\mathrm{H}$, Thorsteinsdottir $\mathrm{U}$, et al. The gene encoding 5 -lipoxygenase activating protein confers risk of myocardial infarction and stroke. Nat Genet 2004; 36: 233-239.

26. Morris JN. Recent history of coronary disease. Lancet 1951 ; 1: 1-7.

27. Morris JN. Recent history of coronary disease. Lancet 1951; 1: $69-73$.

28. Carey MA, Bradbury JA, Seubert JM, Langenbach R, Zeldin DC, Germolec DR. Contrasting effects of cyclooxygenase-1 (COX-1) and COX-2 deficiency on the host response to influenza A viral infection. J Immunol 2005; 175: 6878-6884.

29. Thomas PG, Brown SA, Keating R, Yue W, Morris MY, So J, et al. Hidden epitopes emerge in secondary influenza virusspecific CD8+ T cell responses. J Immunol 2007; 178: 3091-3098. 